\title{
Neuropilin-1 is a host factor for SARS-CoV-2 infection
}

\author{
James L. Daly ${ }^{1 *}$, Boris Simonetti**, Katja Klein ${ }^{2 *}$, Kai-En Chen ${ }^{3}+$, Maia Kavanagh Williamson ${ }^{2}$, Carlos

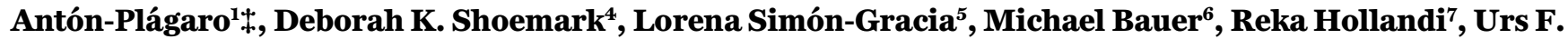 \\ Greber $^{6}$, Peter Horvath ${ }^{7,8}$, Richard B. Sessions ${ }^{1}$, Ari Helenius ${ }^{9}$, Julian A. Hiscox ${ }^{10,11}$, Tambet Teesalu ${ }^{5}$, David A. \\ Matthews ${ }^{2}$, Andrew D. Davidson ${ }^{2}$, Brett M. Collins ${ }^{3}$, Peter J. Cullen' ${ }^{1}$, Yohei Yamauchi ${ }^{2} \uparrow$

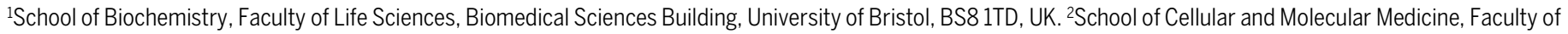 \\ Life Sciences, Biomedical Sciences Building, University of Bristol, BS81TD, UK. IInstitute for Molecular Bioscience, the University of Queensland, St. Lucia, QLD 4072,

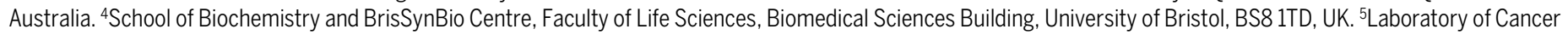 \\ Biology, Institute of Biomedicine and Translational Medicine, University of Tartu, Tartu, Estonia. ${ }^{6}$ Department of Molecular Life Sciences, University of Zurich, \\ Winterthurerstrasse 190, 8057 Zürich, Switzerland. ${ }^{7}$ Synthetic and Systems Biology Unit, Biological Research Centre (BRC), Szeged, Hungary. ${ }^{8}$ Institute for Molecular \\ Medicine Finland, University of Helsinki, Helsinki, Finland. ${ }^{9}$ Institute of Biochemistry, ETH Zurich, Zurich, Switzerland. ${ }^{10}$ Institute of Infection, Veterinary and Ecological \\ Sciences, University of Liverpool, Liverpool, UK. ${ }^{11}$ Singapore Immunology Network, Agency for Science, Technology, and Research, 138648, Singapore. \\ *These authors contributed equally to this work. \\ †Corresponding author. Email: bs13866@bristol.ac.uk (B.S.); pete.cullen@bristol.ac.uk (P.J.C.); yohei.yamauchi@bristol.ac.uk (Y.Y.) \\ $\ddagger$ These authors contributed equally to this work.
}

SARS-CoV-2, the causative agent of COVID-19, uses the viral Spike (S) protein for host cell attachment and entry. The host protease furin cleaves the full-length precursor $S$ glycoprotein into two associated polypeptides: S1 and S2. Cleavage of S generates a polybasic Arg-Arg-Ala-Arg C-terminal sequence on S1, which conforms to a C-end rule (CendR) motif that binds to cell surface Neuropilin-1 (NRP1) and Neuropilin2 (NRP2) receptors. Here, we used X-ray crystallography and biochemical approaches to show that the S1 CendR motif directly bound NRP1. Blocking this interaction using RNAi or selective inhibitors reduced SARS-CoV-2 entry and infectivity in cell culture. NRP1 thus serves as a host factor for SARS-CoV-2 infection and may potentially provide a therapeutic target for COVID-19.

SARS-CoV-2 is the coronavirus responsible for the current COVID-19 pandemic $(1,2)$. A striking difference between the $\mathrm{S}$ protein of SARS-CoV-2 and SARS-CoV is the presence, in the former, of a polybasic sequence motif, RRAR, at the S1/S2 boundary. It provides a cleavage site for a host proprotein convertase, furin (3-5) (fig. S1A). The resulting two proteins, S1 and S2, remain non-covalently associated, with the serine protease TMPRSS2 further priming S2 (6). Furin-mediated processing increases infectivity and affects the tropism of SARS-CoV-2, while furin inhibition diminishes SARS-CoV-2 entry, and deletion of the polybasic site in the $\mathrm{S}$ protein reduces syncytia formation in cell culture $(3-5,7)$.

The $\mathrm{C}$ terminus of the S1 protein generated by furin cleavage has an amino acid sequence $\left({ }^{682} \mathrm{RRAR}^{685}\right)$, that conforms to a $[\mathrm{R} / \mathrm{K}] \mathrm{XX}[\mathrm{R} / \mathrm{K}]$ motif, termed the 'C-end rule' (CendR) (fig. S1B) (8). CendR peptides bind to Neuropilin-1 (NRP1) and NRP2, transmembrane receptors that regulate pleiotropic biological processes, including axon guidance, angiogenesis, and vascular permeability (8-10). To explore the possibility that the SARS-CoV-2 S1 protein may associate with neuropilins we generated a GFP-tagged S1 construct (GFP-S1) (fig. S1C). When expressed in HEK293T cells engineered to express the SARS-CoV-2 receptor ACE2, GFP-S1 immunoprecipitated endogenous NRP1 and ACE2 (Fig. 1A). We transiently co-expressed NRP1-mCherry and either GFP-S1 or GFPS1 $\triangle$ RRAR (a deletion of the terminal ${ }^{682} \mathrm{RRAR}^{685}$ residues) in
HEK293T cells. NRP1 immunoprecipitated the S1 protein, and deletion of the CendR motif reduced this association (Fig. 1B). Comparable binding was also observed with mCherryNRP2, a receptor with high homology to NRP1 (fig. S1, D and E). In both cases, residual binding was observed with the $\triangle$ RRAR mutant indicating an additional CendR-independent association between neuropilins and the S1 protein.

To probe the functional relevance of this interaction, we generated HeLa wild type and NRP1 knock out (KO) cell lines stably expressing ACE2, designated as $\mathrm{HeLa}^{\mathrm{wt}}+\mathrm{ACE} 2$ and $\mathrm{HeLa}^{\mathrm{NRP1KO}}+\mathrm{ACE} 2$ respectively (the level of ACE2 expression was comparable between these lines) (fig. S1F). Using a clinical isolate SARS-CoV-2 (SARS-CoV2/human/Liverpool/REMRQ001/2020), we performed viral infection assays and fixed the cells at 6 and 16 hours post infection (hpi). SARS-CoV-2 infection was reduced in $\mathrm{HeLa}^{\mathrm{NRP1KO}}+\mathrm{ACE} 2$ relative to $\mathrm{HeLa}^{\mathrm{wt}}+\mathrm{ACE} 2$ (Fig. 1C). HeLa cells lacking ACE2 expression were not infected (fig. S1G). In Caco-2 cells, a human colon adenocarcinoma cell line endogenously expressing ACE2 and widely used in COVID-19 studies, the suppression of NRP1 expression by shRNA greatly reduced SARS-CoV-2 infection at both 7 and 16 hpi respectively, whereas that of vesicular stomatitis virus (VSV) pseudotyped with VSV-G was unaffected (Fig. 1D and figs. $\mathrm{S} 1 \mathrm{H}$ and $\mathrm{S} 2 \mathrm{~A}$ ). To determine if NRP1 was required for early virus infection, we established a sequential staining 
procedure using antibodies against SARS-CoV-2 $\mathrm{S}$ and N proteins to distinguish extracellular and intracellular viral particles (fig. S2B). While NRP1 depletion did not affect SARSCoV-2 binding to the Caco-2 cell surface (Fig. 1E), virus uptake was halved in NRP1-depleted cells compared to control cells after 30 min of internalization (Fig. 1F). Thus, NRP1 enhances SARS-CoV-2 entry and infection.

We also observed that SARS-CoV-2-infected $\mathrm{HeLa}^{\mathrm{wt}}+\mathrm{ACE} 2$ cells displayed a multi-nucleated syncytia cell pattern, as reported by others (Fig. 1C) (5). Using an image analysis algorithm and supervised machine learning (fig. S2, C to F) (11), we quantified syncytia of infected $\mathrm{HeLa}^{\mathrm{wt}}+\mathrm{ACE} 2$ and $\mathrm{HeLa}^{\mathrm{NRPIKO}}+\mathrm{ACE} 2$ cells. At $16 \mathrm{hpi}$, the majority of $\mathrm{HeLa}^{\mathrm{wt}}+\mathrm{ACE} 2$ cells formed syncytia, while in $\mathrm{HeLa}^{\mathrm{NRP1KO}}+\mathrm{ACE} 2$ cells this phenotype was reduced (fig. S2G). When infected with a SARS-CoV-2 isolate lacking the furin cleavage site (SARS-CoV-2 $\triangle \mathrm{S} 1 / \mathrm{S} 2$ ) (fig. S1A) the differences in infection and syncytia formation were less pronounced (fig. S2, $\mathrm{H}$ and I). However, a significant decrease in infection of $\mathrm{HeLa}^{\mathrm{NRPIKO}}+\mathrm{ACE} 2$ was still observed at $16 \mathrm{hpi}$, indicating that NRP1 may additionally influence infection through a CendR-independent mechanism (fig. S2H).

The extracellular regions of NRP1 and NRP2 are composed of two CUB domains (a1 and a2), two coagulation factor domains (b1 and b2), and a MAM domain (9). Of these, the b1 domain contains the specific binding site for CendR peptides (fig. S3A) (12). Accordingly, the mCherry-b1 domain of NRP1 immunoprecipitated GFP-S1, and a shortened GFPS1 construct spanning residues 493-685 (figs. S1C and S3B). Isothermal titration calorimetry (ITC) established that the b1 domain of NRP1 directly bound a synthetic S1 CendR peptide $\left({ }^{679} \mathrm{NSPRRAR}^{685}\right)$ with an affinity of $20.3 \mu \mathrm{M}$ at $\mathrm{pH} 7.5$, which was enhanced to $13.0 \mu \mathrm{M}$ at pH 5.5 (Fig. 2A). Binding was not observed to a S1 CendR peptide in which the C-terminal arginine was mutated to alanine $\left({ }^{679} \mathrm{NSPRRAA}^{685}\right)$ (Fig. 2A). We co-crystallized the NRP1 b1 domain in complex with the S1 CendR peptide (Fig. 2B). The resolved $2.35 \AA$ structure revealed 4 molecules of b1 with electron density of the S1 CendR peptide clearly visible in the asymmetric unit (fig. S3C). S1 CendR peptide binding displayed remarkable similarity to the previously solved structure of NRP1 b1 domain in complex with its endogenous ligand VEGF- $\mathrm{A}_{164}$ (Fig. 2B and fig. S3D) (12). The key residues responsible for contacting the C-terminal R685 of the CendR peptide - Y297, W301, T316, D320, S346, T349 and Y353 - are almost identical between the two structures (Fig. 2B and fig. S3D). The R682 and R685 sidechains together engage NRP1 via stacked cation- $\pi$ interactions with NRP1 side chains of Y297 and Y353. By projecting these findings onto the structure of the NRP1 ectodomain, the b1 CendR binding pocket appears to be freely accessible to the S1 CendR peptide (fig. S3E) (13).

Site-directed mutagenesis of the S1 R685 residue to aspartic acid drastically reduced GFP-S1 ${ }^{493-685}$ immunoprecipitation by mCherry-b1, confirming the critical role of the Cterminal arginine (Fig. 2C). Mutagenesis of the T316 residue within the mCherry-b1 domain of NRP1 to arginine also reduced association with GFP-S1 ${ }^{493-685}$, consistent with its inhibitory impact on VEGF-A $\mathrm{A}_{164}$ binding (12) (Fig. 2D). Accordingly, incubation of mCherry-b1 with VSV particles pseudotyped with trimeric $\mathrm{S}$ resulted in immunoprecipitation of processed forms of S1, which was dependent on the T316 residue (fig. S3F). Next, we transiently expressed either GFP, full length NRP1 wt-GFP or full length NRP1-GFP harboring the T316R mutation in $\mathrm{HeLa}^{\mathrm{NRP1KO}}+\mathrm{ACE} 2$ cells. GFP expression and ACE2 expression levels were comparable and both constructs retained similar cell surface localization (fig. S3, G and $\mathrm{H}$ ). SARS-CoV-2 infection was significantly enhanced in cells expressing NRP1 wt-GFP compared to GFP control, whereas it was not enhanced in cells expressing the T316R mutant (Fig. 2E). Thus, the SARS-CoV-2 S1 CendR and NRP1 interaction promotes infection.

To establish the functional relevance of the S1 CendRNRP1 interaction, we screened monoclonal antibodies (mAb\#1, mAb\#2, mAb\#3) raised against the NRP1 b1b2 ectodomain. All three bound to the NRP1 b1b2 domain, displayed staining by immunofluorescence in NRP1-expressing PPC-1 (human primary prostate cancer) cells but not in M21 (human melanoma) cells that do not express NRP1 (fig. S4A) (8), and stained the extracellular domain of NRP1-GFP expressed in cells (fig. S4B). Of these antibodies, $\mathrm{mAb} \# 3$, and to a lesser

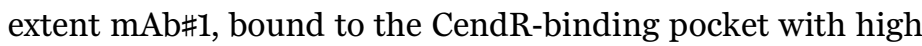
specificity, as defined by reduced ability to bind to a b1b2 mutant that targets residues (S346, E348, T349) at the opening of the binding pocket (Fig. 3A) (12). Incubation of Caco-2 cells with mAbs\#1 and 3, reduced SARS-CoV-2 infection compared to a control mAb targeting avian influenza A virus (H11N3) hemagglutinin (Fig. 3B). Consistent with this, mAb\#3 inhibited binding of GFP-S1 ${ }^{493-685}$ and mCherry-b1 (Fig. 3C). As a comparison, Caco-2 and Calu-3 cells were incubated with soluble ACE2, which inhibited SARS-CoV-2 infection in both cases (fig. S4C).

Next, we turned to the small molecule EG00229, a selective NRP1 antagonist that binds the b1 CendR binding pocket and inhibits VEGF-A binding (Fig. 3D) (14). ITC established that EG00229 bound to the NRP1 b1 domain with a $\mathrm{K}_{\mathrm{d}}$ of 5.1 and $11.0 \mu \mathrm{M}$ at pH 7.5 and 5.5 respectively (Fig. 3E). EG00229 inhibited the direct binding between b1 and the S1 CendR peptide, and the immunoprecipitation of GFP-S1 ${ }^{493-685}$ by mCherry-b1 (Fig. 3E and fig. S4D). Finally, incubation of Caco-2 cells with EG00229 reduced the efficiency of SARSCoV-2 infection at 7 and 16 hpi (Fig. 3F). Thus, the SARS-CoV2 interaction with NRP1 can be targeted to reduce viral infectivity in relevant human cell lines (fig. S5).

Cell entry of SARS-CoV-2 depends on priming by host cell 
proteases $(5,6,15)$. Our data indicate that a component of SARS-CoV-2 S protein binding to cell surface neuropilins occurs via the S1 CendR motif generated by the furin cleavage of $\mathrm{S} 1 / \mathrm{S} 2$. While not affecting cell surface attachment, this interaction promotes entry and infection by SARS-CoV-2 in physiologically relevant cell lines widely used in the study of COVID-19. The molecular basis for the effect is unclear, but neuropilins are known to mediate the internalization of CendR ligands through an endocytic process resembling macropinocytosis, $(8,16,17)$. Interestingly, gene expression analysis has revealed an up-regulation of NRP1 and NRP2 in lung tissue from COVID-19 patients (18). A SARS-CoV-2 virus with a natural deletion of the $\mathrm{S} 1 / \mathrm{S} 2$ furin cleavage site demonstrated attenuated pathogenicity in hamster models (19). NRP1 binding to the CendR peptide in S1 is thus likely to play a role in the increased infectivity of SARS-CoV-2 compared with SARS-CoV. The ability to target this specific interaction may provide a route for COVID-19 therapies.

\section{REFERENCES AND NOTES}

1. WHO Coronavirus disease, 2019 (COVID-19) Weekly Epidemiological Update - 31 August 2020. https://www.who.int/docs/defaultsource/coronaviruse/situation-reports/20200831-weekly-epi-update3.pdf?sfvrsn $=$ d7032a2a 4

2. E. Dong, H. Du, L. Gardner, An interactive web-based dashboard to track COVID-19 in real time. Lancet Infect. Dis. 20, 533-534 (2020). doi:10.1016/S14733099(20)30120-1 Medline

3. D. Wrapp, N. Wang, K. S. Corbett, J. A. Goldsmith, C.-L. Hsieh, O. Abiona, B. S. Graham, J. S. McLellan, Cryo-EM structure of the 2019-nCoV spike in the prefusion conformation. Science 367, 1260-1263 (2020). doi:10.1126/science. abb2507 Medline

4. A. C. Walls, Y.-J. Park, M. A. Tortorici, A. Wall, A. T. McGuire, D. Veesler, Structure, Function, and Antigenicity of the SARS-CoV-2 Spike Glycoprotein. Cell 181, 281292.e6 (2020). doi:10.1016/i.cell.2020.02.058 Medline

5. M. Hoffmann, H. Kleine-Weber, S. Pöhlmann, A multibasic cleavage site in the spike protein of SARS-CoV-2 is essential for infection of human lung cells. Mol. Cell 78, 779-784.e5 (2020). doi:10.1016/i.molcel.2020.04.022 Medline

6. M. Hoffmann, H. Kleine-Weber, S. Schroeder, N. Krüger, T. Herrler, S. Erichsen, T. S. Schiergens, G. Herrler, N.-H. Wu, A. Nitsche, M. A. Müller, C. Drosten, S. Pöhlmann, SARS-CoV-2 cell entry depends on ACE2 and TMPRSS2 and is blocked by a clinically proven protease inhibitor. Cell 181, 271-280.e8 (2020). doi:10.1016/i.cell.2020.02.052 Medline

7. J. Shang, Y. Wan, C. Luo, G. Ye, Q. Geng, A. Auerbach, F. Li, Cell entry mechanisms of SARS-CoV-2. Proc. Natl. Acad. Sci. U.S.A. 117, 11727-11734 (2020). doi:10.1073/pnas.2003138117 Medline

8. T. Teesalu, K. N. Sugahara, V. R. Kotamraju, E. Ruoslahti, C-end rule peptides mediate neuropilin-1-dependent cell, vascular, and tissue penetration. Proc. Natl. Acad. Sci. U.S.A. 106, 16157-16162 (2009). doi:10.1073/pnas.0908201106 Medline

9. H. F. Guo, C. W. Vander Kooi, Neuropilin functions as an essential cell surface receptor. J. Biol. Chem. 290, 29120-29126 (2015). doi:10.1074/jbc.R115.687327 Medline

10. A. Plein, A. Fantin, C. Ruhrberg, Neuropilin regulation of angiogenesis, arteriogenesis, and vascular permeability. Microcirculation 21, 315-323 (2014). doi:10.1111/micc.12124 Medline

11. R. Hollandi, A. Szkalisity, T. Toth, E. Tasnadi, C. Molnar, B. Mathe, I. Grexa, J. Molnar, A. Balind, M. Gorbe, M. Kovacs, E. Migh, A. Goodman, T. Balassa, K. Koos, W. Wang, J. C. Caicedo, N. Bara, F. Kovacs, L. Paavolainen, T. Danka, A. Kriston, A. E. Carpenter, K. Smith, P. Horvath, nucleAlzer: A parameter-free deep learning framework for nucleus segmentation using image style transfer. Cell Syst. 10 , 453-458.e6 (2020). doi:10.1016/i.cels.2020.04.003
12. M. W. Parker, P. Xu, X. Li, C. W. Vander Kooi, Structural basis for selective vascular endothelial growth factor-A (VEGF-A) binding to neuropilin-1. J. Biol. Chem. 287, 11082-11089 (2012). doi:10.1074/ibc.M111.331140 Medline

13. B. J. Janssen, T. Malinauskas, G. A. Weir, M. Z. Cader, C. Siebold, E. Y. Jones, Neuropilins lock secreted semaphorins onto plexins in a ternary signaling complex. Nat. Struct. Mol. Biol. 19, 1293-1299 (2012). doن:10.1038/nsmb.2416 Medline

14. A. Jarvis, C. K. Allerston, H. Jia, B. Herzog, A. Garza-Garcia, N. Winfield, K. Ellard, R. Aqil, R. Lynch, C. Chapman, B. Hartzoulakis, J. Nally, M. Stewart, L. Cheng, M. Menon, M. Tickner, S. Djordjevic, P. C. Driscoll, I. Zachary, D. L. Selwood, Small molecule inhibitors of the neuropilin-1 vascular endothelial growth factor A (VEGFA) interaction. J. Med. Chem. 53, 2215-2226 (2010). doi:10.1021/im901755g Medline

15. J. K. Millet, G. R. Whittaker, Physiological and molecular triggers for SARS-CoV membrane fusion and entry into host cells. Virology 517, 3-8 (2018). doi:10.1016/i.virol.2017.12.015 Medline

16. M. Simons, E. Gordon, L. Claesson-Welsh, Mechanisms and regulation of endothelial VEGF receptor signalling. Nat. Rev. Mol. Cell Biol. 17, 611-625 (2016). doi:10.1038/nrm.2016.87 Medline

17. H. B. Pang, G. B. Braun, T. Friman, P. Aza-Blanc, M. E. Ruidiaz, K. N. Sugahara, T. Teesalu, E. Ruoslahti, An endocytosis pathway initiated through neuropilin-1 and regulated by nutrient availability. Nat. Commun. 5, 4904 (2014). doi: $10.1038 /$ ncomms 5904 Medline

18. M. Ackermann, S. E. Verleden, M. Kuehnel, A. Haverich, T. Welte, F. Laenger, A. Vanstapel, C. Werlein, H. Stark, A. Tzankov, W. W. Li, V. W. Li, S. J. Mentzer, D. Jonigk, Pulmonary vascular endothelialitis, thrombosis, and angiogenesis in Covid-19. N. Engl. J. Med. 383, 120-128 (2020). doi:10.1056/NEJMoa2015432 Medline

19. S.-Y. Lau, P. Wang, B. W.-Y. Mok, A. J. Zhang, H. Chu, A. C.-Y. Lee, S. Deng, P. Chen, K.-H. Chan, W. Song, Z. Chen, K. K.-W. To, J. F.-W. Chan, K.-Y. Yuen, H. Chen, Attenuated SARS-CoV-2 variants with deletions at the S1/S2 junction. Emerg. Microbes Infect. 9, 837-842 (2020). doi:10.1080/222221751,2020.1756700 Medline

20. A. D. Davidson, M. K. Williamson, S. Lewis, D. Shoemark, M. W. Carroll, K. J. Heesom, M. Zambon, J. Ellis, P. A. Lewis, J. A. Hiscox, D. A. Matthews, Characterisation of the transcriptome and proteome of SARS-CoV-2 reveals a cell passage induced in-frame deletion of the furin-like cleavage site from the spike glycoprotein. Genome Med. 12, 68 (2020). doi:10.1186/s13073-020-00763-0 Medline

21. M. Berger Rentsch, G. Zimmer, A vesicular stomatitis virus replicon-based bioassay for the rapid and sensitive determination of multi-species type I interferon. PLOS ONE 6, e25858 (2011). doi:10.1371/journal.pone.0025858 Medline

22. K. Smith, Y. Li, F. Piccinini, G. Csucs, C. Balazs, A. Bevilacqua, P. Horvath, CIDRE: An illumination-correction method for optical microscopy. Nat. Methods 12, 404406 (2015). doi:10.1038/nmeth.3323 Medline

23. Ronneberger, O., Fischer, P., and Brox. T. U-net: Convolutional networks for biomedical image segmentation. Medical Image Computing and Computerassisted intervention. 234-241, Springer International Publishing (2015).

24. F. Piccinini, T. Balassa, A. Szkalisity, C. Molnar, L. Paavolainen, K. Kujala, K. Buzas, M. Sarazova, V. Pietiainen, U. Kutay, K. Smith, P. Horvath, Advanced cell classifier: User-friendly machine-learning-based software for discovering phenotypes in high-content imaging data. Cell Syst. 4, 651-655.e5 (2017) doi:10.1016/i.cels.2017.05.012 Medline

25. B. A. Appleton, P. Wu, J. Maloney, J. Yin, W.-C. Liang, S. Stawicki, K. Mortara, K. K. Bowman, J. M. Elliott, W. Desmarais, J. F. Bazan, A. Bagri, M. Tessier-Lavigne, A. W. Koch, Y. Wu, R. J. Watts, C. Wiesmann, Structural studies of neuropilin/antibody complexes provide insights into semaphorin and VEGF binding. EMBO J. 26, 4902-4912 (2007). doi:10.1038/sj.emboj.7601906 Medline

26. C. W. Vander Kooi, M. A. Jusino, B. Perman, D. B. Neau, H. D. Bellamy, D. J. Leahy, Structural basis for ligand and heparin binding to neuropilin B domains. Proc. Natl. Acad. Sci. U.S.A. 104, 6152-6157 (2007). doi:10.1073/pnas.0700043104 Medline

27. B. G. Dorner, S. Steinbach, M. B. Hüser, R. A. Kroczek, A. Scheffold, Single-cell analysis of the murine chemokines MIP- $1 \alpha$, MIP- $1 \beta, \quad$ RANTES and ATAC/lymphotactin by flow cytometry. J. Immunol. Methods 274, 83-91 (2003). 
doi:10.1016/S0022-1759(02)00498-2 Medline

28. W. Kabsch, XDS. Acta Crystallogr. D Biol. Crystallogr. 66, 125-132 (2010). doi:10.1107/S0907444909047337 Medline

29. P. R. Evans, G. N. Murshudov, How good are my data and what is the resolution? Acta Crystallogr. D Biol. Crystallogr. 69, 1204-1214 (2013). doi:10.1107/S0907444913000061 Medline

30. A. J. McCoy, R. W. Grosse-Kunstleve, P. D. Adams, M. D. Winn, L. C. Storoni, R. J. Read, Phaser crystallographic software. J. Appl. Crystallogr. 40, 658-674 (2007). doi:10.1107/S0021889807021206 Medline

31. P. D. Adams, P. V. Afonine, G. Bunkóczi, V. B. Chen, I. W. Davis, N. Echols, J. J. Headd, L.-W. Hung, G. J. Kapral, R. W. Grosse-Kunstleve, A. J. McCoy, N. W. Moriarty, R. Oeffner, R. J. Read, D. C. Richardson, J. S. Richardson, T. C. Terwilliger, P. H. Zwart, PHENIX: A comprehensive Python-based system for macromolecular structure solution. Acta Crystallogr. D Biol. Crystallogr. 66, 213221 (2010). doi:10.1107/S0907444909052925 Medline

32. V. B. Chen, W. B. Arendall 3rd, J. J. Headd, D. A. Keedy, R. M. Immormino, G. J. Kapral, L. W. Murray, J. S. Richardson, D. C. Richardson, MolProbity: All-atom structure validation for macromolecular crystallography. Acta Crystallogr. D Biol. Crystallogr. 66, 12-21 (2010). doi:10.1107/S0907444909042073 Medline

33. L. Holm, P. Rosenström, Dali server: Conservation mapping in 3D. Nucleic Acids Res. 38, W545-9 (2010). doi:10.1093/nar/gkq366 Medline

\section{ACKNOWLEDGMENTS}

We thank the Bristol Synthetic Biology Centre and the Advanced Computing Research Centre for provision of HPC (Bluegem), and the University of Bristol Wolfson Bioimaging Facility. We thank the University of Queensland Remote Operation Crystallisation and X-ray facility (UQ-ROCX) and the staff for their support with the crystallization experiments, and the staff of the Australian Synchrotron for assistance with X-ray diffraction data collection. Funding: JLD was supported by a Wellcome Trust studentship from the Dynamic Molecular Cell Biology Ph.D. program (203959/Z/16/Z), CAP was supported by Beca Fundación Ramón Areces Estudios Postdoctorales en el Extranjero and MKW was supported by a MRC grant (MR/R020566/1) awarded to ADD. This project has received funding from the MRC (MR/P018807/1), Wellcome Trust (104568/Z/14/2), Lister Institute of Preventive Medicine, and Elizabeth Blackwell Institute for Health Research Rapid Response Call (COVID-19) awarded to PJC, the European Research Council under the European Union's Horizon 2020 research and innovation program (No 856581 - CHUbVi), and from MRCAMED (MR/T028769/1) awarded to YY, the Swiss National Science Foundation and Kanton Zurich awarded to UFG. BMC is supported by an Australian National Health and Medical Research Council (NHMRC) Senior Research Fellowship (APP1136021) and Project Grant (APP1156493), and the United States Food and Drug Administration grant number HHSF223201510104C 'Ebola Virus Disease: correlates of protection, determinants of outcome and clinical management' amended to incorporate urgent COVID-19 studies awarded to JAH, ADD and DAM. RH and PH acknowledge support from the LENDULET-BIOMAG Grant (2018-342), from H2020-discovAIR (874656), and from Chan Zuckerberg Initiative, Seed Networks for the HCA-DVP. TT was supported by the European Regional Development Fund (Project No. 2014-2020.4.01.15-0012), by European Research Council grant GLIOGUIDE and Estonian Research Council (grants PRG230 and EAG79, to T.T.). Author contributions: JLD, BS, AH, PJC and YY conceived the study. JLD, BS, KK and YY performed most of the experiments. KK, MKW, DAM and ADD performed all work with infectious SARS-CoV-2 supervised by ADD. MKW and ADD isolated SARS-CoV-2 strains used for the work. KC, CAP, MB, LSG, UFG, KK, RBS, DKS, JAH and TT did experimental work and/or provided essential reagents. $\mathrm{RH}$ and $\mathrm{PH}$ performed image analysis. $\mathrm{BS}$, ADD, BMC, PJC and YY supervised the research. JLD, BS, ADD, PJC and YY wrote the manuscript and made the figures. All authors read and approved the final manuscript. Competing onterests: T. Teesalu is an inventor of patents on CendR peptides and shareholder of Cend Therapeutics Inc., a company that holds a license for the CendR peptides and is developing the peptides for cancer therapy. J. Hiscox is a member of the Department of Health, New and Emerging Respiratory Virus Threats Advisory Group (NERVTAG) and the Department of Health, Testing Advisory Group. U. Greber is a consultant to F. Hoffmann-La Roche Ltd, Switzerland. All other authors declare no competing interests. Data and materials availability: Coordinates and structure factors for the NRP1 b1 S1 CendR peptide complex have been deposited at the Protein Data Bank (PDB) with accession code 7JJC. All other data are available in the manuscript or the supplementary material. This work is licensed under a Creative Commons Attribution 4.0 International (CC BY 4.0) license, which permits unrestricted use, distribution, and reproduction in any medium, provided the original work is properly cited. To view a copy of this license, visit

https://creativecommons.org/licenses/by/4.0/. This license does not apply to figures/photos/artwork or other content included in the article that is credited to a third party; obtain authorization from the rights holder before using such material.

\section{SUPPLEMENTARY MATERIALS}

science.sciencemag.org/cgi/content/full/science.abd3072/DC1

Materials and Methods

Figs. S1 to S5

Tables S1 to S3

References (20-33)

MDAR Reproducibility Checklist

14 June 2020; accepted 12 October 2020

Published online 20 October 2020

10.1126/science.abd3072 

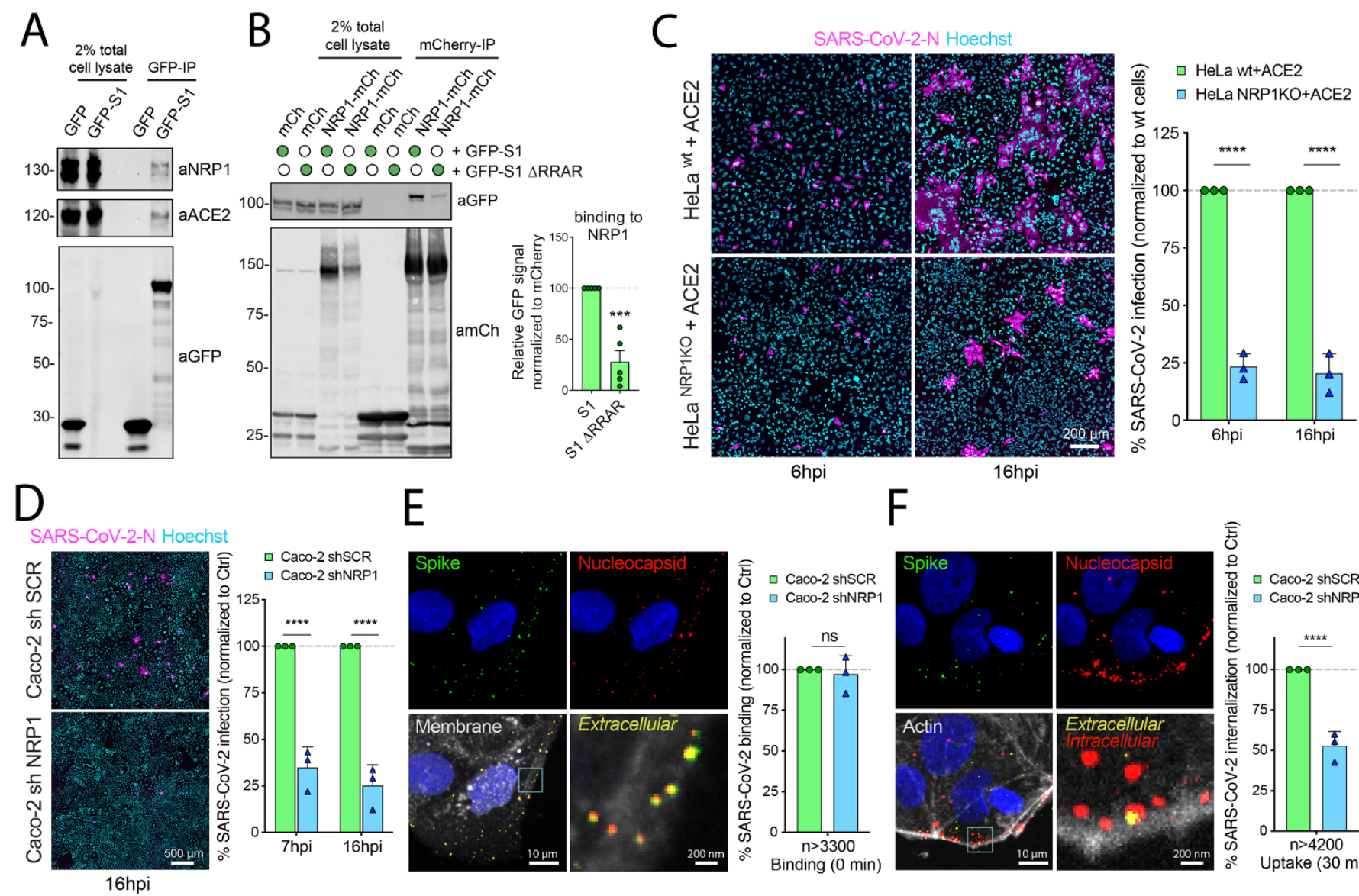

Fig. 1. NRP1 Interacts with S1 and enhances SARS-CoV-2 infection. (A) HEK293T cells transduced to express ACE2 were transfected to express GFP or GFP-tagged S1 and lysed after 24h. The lysates were subjected to GFP-nanotrap and the immune-isolates were blotted for ACE2 and NRP1 (N=3). (B) HEK293T cells were cotransfected to express GFP-tagged S1 or GFP-S1 $\triangle$ RRAR and mCherry or mCherry-tagged NRP1 and subjected to GFP-nanotrap ( $N=5$ ). Two-tailed unpaired t-test; $P=0.0002$. (C) HeLawt $+A C E 2$ and HeLa ${ }^{\mathrm{NRP1}} \mathrm{kO}+\mathrm{ACE} 2$ cells were infected with SARS-CoV-2. Cells were fixed at 6 or $16 \mathrm{hpi}$ and stained for N protein (magenta) and Hoechst (cyan), and virus infectivity was quantified ( $N=3$ ). Two-tailed unpaired t-test; $P=0.00002$ and 0.00088 . Scale bar $=200 \mu \mathrm{m}$. (D) Caco-2 cells expressing shRNA against NRP1 or a non-targeting control (SCR) were infected with SARS-CoV-2 and fixed at 7 or $16 \mathrm{hpi}$. The cells were stained for N protein (magenta) and Hoechst (cyan), and infectivity was quantified ( $\mathrm{N}=3$ ). Two-tailed unpaired t-test; $\mathrm{P}=0.0005$ and 0.00032 . Scale bar $=500 \mu \mathrm{m}$. (E) Caco-2 shSCR or shNRP1 cells were inoculated with MOI=50 of SARS-CoV-2 and incubated in the cold for $60 \mathrm{~min}$, and fixed. A two-step antibody staining procedure was performed using anti-S and -N Abs to distinguish external (green) and total (red) virus particles, and the binding of particles per cell was quantified for over 3300 particles per condition ( $N=3$ ). Two-tailed unpaired t-test; $P=0.6859$. ( $F$ ) Caco-2 shSCR or shNRP1 cells were bound with SARS-CoV-2 as in (E), followed by incubation at $37^{\circ} \mathrm{C}$ for $30 \mathrm{~min}$. The cells were fixed and stained as in (E). Viral uptake was quantified for over 4200 particles per condition $(\mathrm{N}=3)$. Two-tailed unpaired t-test; $P=0.00079$. Scale bars for $(E)$ and $(F)=10 \mu \mathrm{m}$ and $200 \mathrm{~nm}$ (zoom panels). The square regions were zoomed in. The bars, error bars, circles and triangles represent the mean, SEM (B) and SD (C-F), individual data points, respectively. $* \mathrm{P}<0.05, * * \mathrm{P}<0.01, * * * \mathrm{P}<0.001, * * * * \mathrm{P}<0.0001$. 
A

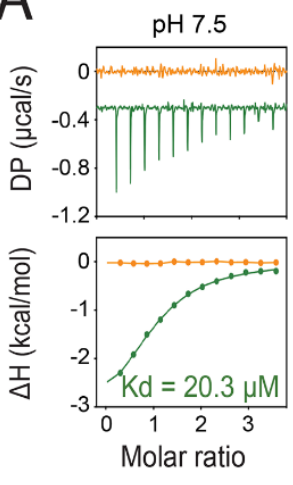

S1 CendR peptide:
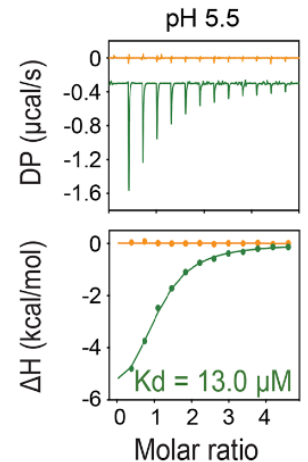

Native
Molar ratio

$\square$ R685A mutant
B

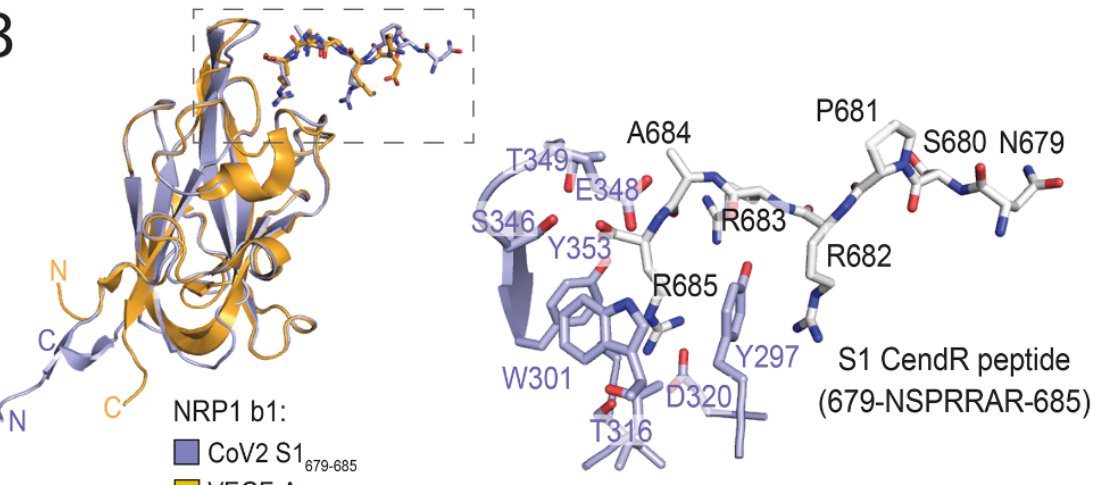

NRP1 b1
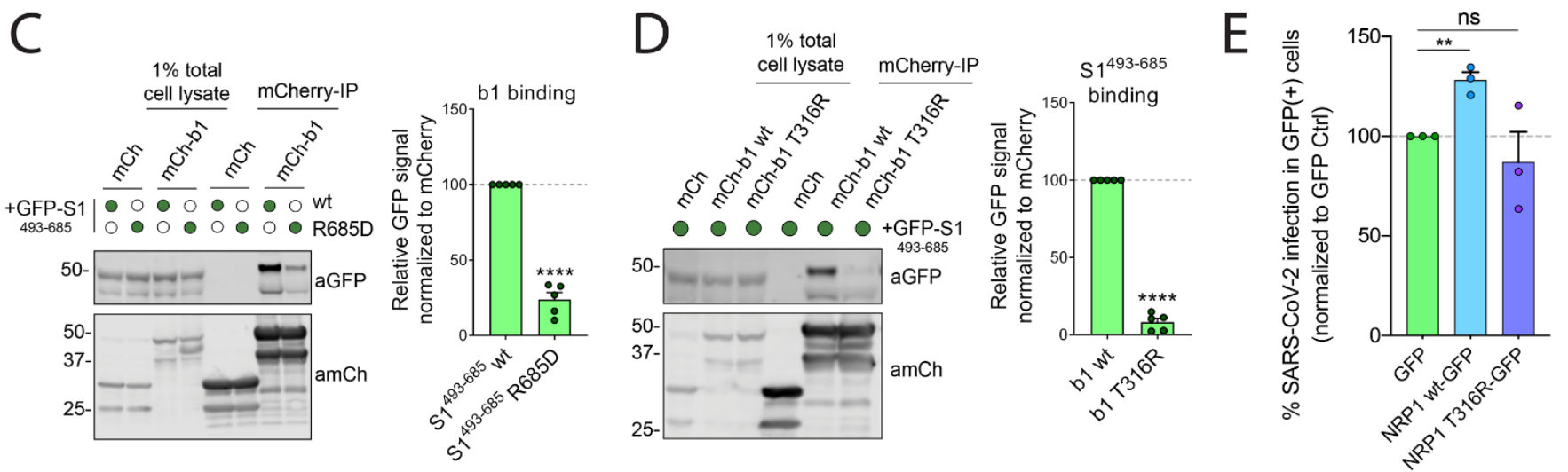

Fig. 2. Molecular basis for CendR binding of SARS-CoV-2 S1 with NRP1. (A) Binding of NRP1 b1 with native (green line) and mutant (orange line) form of S1 CendR peptide (corresponding to residues 679-685) by ITC at two different $\mathrm{pH}$ conditions $(\mathrm{N}=3)$. All ITC graphs represents the integrated and normalized data fit with 1-to-1 ratio binding. (B) Left: NRP1 b1 - S1 CendR peptide complex superposed with NRP1 b1 - VEGF-A fusion complex (PDB ID: 4DEQ). Bound peptides are shown in stick representation. RMSD = root mean square deviation. Right: Enlarged view highlighting the binding of S1 CendR peptide b1. Key binding residues on b1 are shown in stick representation. (C). HEK293T cells were co-transfected with combinations of GFP-tagged S1 ${ }^{493-685}$ and S1 $1^{493-685}$ R685D, and mCherry or mCherry-NRP1 b1, and subjected to mCherry-nanotrap ( $\mathrm{N}=5$ ). Two-tailed unpaired ttest; $\mathrm{P}<0.0001$. (D). HEK293T cells were co-transfected with combinations of GFP-tagged S1 ${ }^{493-685}$ and mCherry, mCherry-NRP1 b1 or mCherry-NRP1 b1 T316R mutant and subjected to mCherry-nanotrap $(\mathrm{N}=5)$. Two-tailed unpaired t-test; $P<0.0001$. (E) HeLa ${ }^{\text {NRP1KO }}+$ ACE2 cells transfected with GFP, NRP1 wt-GFP or NRP1 T316R-GFP constructs were infected $24 \mathrm{~h}$ later with SARS-CoV-2. At $16 \mathrm{hpi}$ the cells were fixed and stained for SARS-CoV-2-N, and viral infection quantified in the GFP-positive subpopulation of cells $(\mathrm{N}=3)$. The percentage of infection was normalized to that of GFP-transfected cells. Two-tailed unpaired t-test; $p=0.002$. The bars, error bars and circles represent the mean, SEM (C-D) and SD (E), individual data points, respectively. $* P<0.05$, $* * \mathrm{P}<0.01, * * * \mathrm{P}<0.001, * * * * \mathrm{P}<0.0001$. 

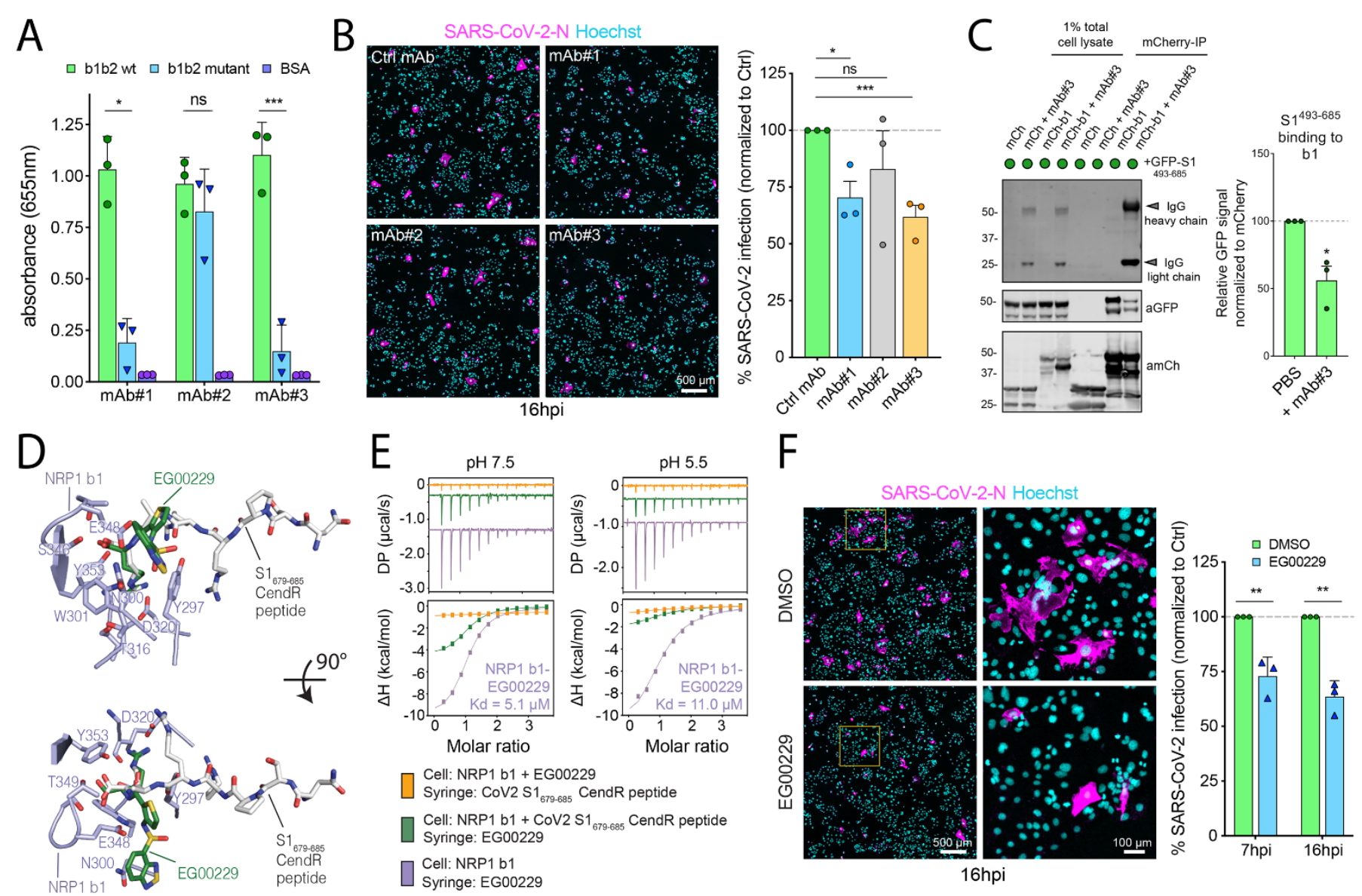

Fig. 3. Selective inhibition of the S1-NRP1 interaction reduces SARS-CoV-2 infection. (A) ELISA of anti-NRP1 monoclonal antibodies ( $m A b \# 1, m A b \# 2, m A b \# 3$ ) at $3 \mu \mathrm{g} / \mathrm{mL}$ using plates coated with NRP1 b1b2 wild type, b1b2 mutant (S346A, E348A, T349A) or BSA, used as control $(\mathrm{N}=3)$. Binding is represented as arbitrary units of absorbance at $655 \mathrm{~nm}$. Two-tailed unpaired t-test; $\mathrm{P}=0.0207,0.2430,0.0007$. (B) Cells were pre-treated with $100 \mu \mathrm{g} / \mathrm{mL}$ of anti-H11N3 (Ctrl) mAb, mAb\#1, 2 or 3 for $1 \mathrm{~h}$ prior to infection with SARS-CoV-2. Cells were fixed at 16 hpi and stained for $\mathrm{N}$ protein (magenta) and Hoechst (cyan) ( $\mathrm{N}=3$ ). Two-tailed unpaired t-test; $\mathrm{P}=0.015,0.36,0.0003$. Scale bar $=500 \mu \mathrm{m}$. (C) HEK293T cells were co-transfected with combinations of mCherry or mCherry-b1 and GFP-tagged S1493-685 and subjected to mCherry-nanotrap with or without coincubation with mAb\#3 (N=3). Two-tailed unpaired t-test; $P=0.0143$. (D) NRP1 b1 - S1 CendR peptide complex superimposed with NRP1 b1 - EG00229 inhibitor complex (PDB ID:3197). Key binding residues on b1, bound peptides and EG00229 are shown in stick representation. (E) ITC analysis of EG00229 binding to b1 domain of NRP1 at two different pH conditions. Pre-incubation with EG00229 blocks S1 CendR peptide binding (orange line), and the CendR peptide can reduce binding of EG00229 (green line). ( $N=3$ ). All ITC graphs represents the integrated and normalized data fit with 1-to-1 ratio binding. (F). Cells were pre-treated with $100 \mu \mathrm{M}$ of EG00229 or DMSO prior to infection with SARS-CoV-2. Cells were fixed at 7 and $16 \mathrm{hpi}$ and stained for N protein (magenta) and Hoechst (cyan) $(\mathrm{N}=3)$. The square region was zoomed in. Scale bars=500 $\mu \mathrm{m}$ and $100 \mu \mathrm{m}$ (zoom panel). Two-tailed unpaired t-test; $P=0.0059$ and 0.0013 . The bars, error bars, circles and triangles represent the mean, SEM (C) and SD (A, B, F) and individual data points, respectively. $* \mathrm{P}<0.05, * * \mathrm{P}<0.01, * * * \mathrm{P}<0.001$, $* * * * \mathrm{P}<0.0001$. 